\title{
ent-Homoabyssomicins A and B, Two New Spirotetronate Metabolites from Streptomyces sp. Ank 210
}

Muna Ali Abdalla, Prem P. Yadav, Birger Dittrich, Anja Schüffler, Hartmut Laatsch*

\author{
SUPPLEMENTARY INFORMATION
}

General Experimental Procedures. The optical rotations were measured on a Perkin-Elmer polarimeter (model 241). IR spectra were recorded on a Perkin-Elmer 1600 Series FT-IR spectrometer with $\mathrm{KBr}$ pellets. NMR spectra were measured on a Varian Inova $600(599.740 \mathrm{MHz})$ and a Varian Unity $300(300.145 \mathrm{MHz}) \mathrm{spec}-$ trometer. ESI mass spectra were recorded on a Quattro Triple Quadrupol mass spectrometer, with a Finnigan TSQ 7000 with nano-ESI API ion source. HRESI mass spectra were measured on a Micromass LCT mass spectrometer coupled with a HP 1100 HPLC and a diode array detector. Data collection of diffraction intensities was performed on a Bruker SMART 6000 area detector diffractometer with $\mathrm{CuK}_{\alpha}$ radiation generated from a $5 \mathrm{~kW}$ rotating anode. Flash chromatography was carried out on silica gel (230-400 mesh). Thin-layer chromatography (TLC) was performed on Polygram SIL G/UV 254 (Macherey-Nagel \& Co.). $R_{f}$ values were measured on Polygram SIL G/UV 254 (Macherey-Nagel \& Co.). Size exclusion chromatography was done on Sephadex LH-20 (Lipophilic Sephadex; Amersham Biosciences, Ltd., purchased from Sigma-Aldrich Chemie).

\section{Isolation and Taxonomy}

The Streptomyces strain ANK 210 has been derived from a forest soil sample (Kaiserslautern) and was isolated on YMG agar at room temperature (YMG agar: 2 $\mathrm{g} / \mathrm{L}$ yeast extract, $5 \mathrm{~g} / \mathrm{L}$ malt extract, $5 \mathrm{~g} / \mathrm{L}$ glucose, $15 \mathrm{~g} / \mathrm{L}$ agar, $30 \mathrm{mg} / \mathrm{L}$ cycloheximide). Its almost complete 16S rRNA gene sequence (GenBank Accession Nr. HQ124331) showed similarities (98.8\%) to Streptomyces miharaensis NBRC 13791 (GenBank Accession Nr. AB184482). A voucher specimen of Streptomyces 
sp. strain Ank 210 was deposited in the culture collection at the Institute of Organic and Biomolecular Chemistry, Göttingen, Germany.

\section{Fermentation and Isolation}

Well-grown agar plates were used to inoculate 100 of 1 L Erlenmeyer flasks each containing $250 \mathrm{ml}$ of $\mathrm{M}_{2}{ }^{+}$medium. The culture was incubated on the shaker ( $95 \mathrm{rpm}$ ) at $28{ }^{\circ} \mathrm{C}$ for 7 days. The resulting brown culture broth was mixed with $c a .1 \mathrm{~kg}$ diatomaceous earth (Celite) and filtered by means of a filter press to afford the aqueous filtrate and a mycelial fraction. The aqueous fraction was extracted with Amberlite XAD-16 resin and eluted with $\mathrm{MeOH}$. The $\mathrm{MeOH}$ eluate was evaporated till to the aqueous residue and the latter one extracted with EtOAc.

The mycelium was three times extracted with EtOAc followed by acetone $(1 \times)$ and the EtOAc and acetone phases were evaporated to dryness. As the three crude extracts showed no differences on TLC, they were combined to obtain $5.8 \mathrm{~g}$ of a brown residue. The crude extract was dissolved in methanol, defatted with cyclohexane and subjected to silica gel column chromatography using $\mathrm{CH}_{2} \mathrm{Cl}_{2} / \mathrm{MeOH}$ gradient (column $3 \times 60 \mathrm{~cm}, 0$ to $20 \% \mathrm{MeOH}$ ). Fraction FII was purified on Sephadex LH-20 using $\mathrm{MeOH}$ followed by $\mathrm{RP}-18$ using $\mathrm{MeOH} / \mathrm{H}_{2} \mathrm{O}$ gradient (10 to $50 \% \mathrm{MeOH}$ ) to deliver $12 \mathrm{mg}$ ent-homoabyssomicins A (1) and $5 \mathrm{mg} \mathrm{B}$ (2). In a similar way, fraction FIII was purified on Sephadex LH-20 using MeOH followed by silica gel (cyclohexane/EtOAc, gradient 0 to $100 \%$ EtOAc) afforded indole-3-carboxylic acid, $4.7 \mathrm{mg}$ 2-hydroxy-1-(4-hydroxy-3-methoxy-phenyl)-ethanone, and $17 \mathrm{mg}$ polypro-

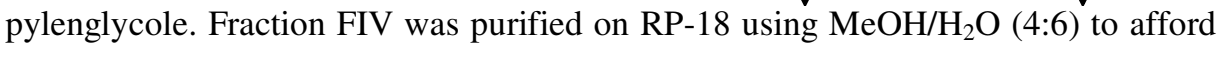
$6.3 \mathrm{mg}$ benadrostin. 


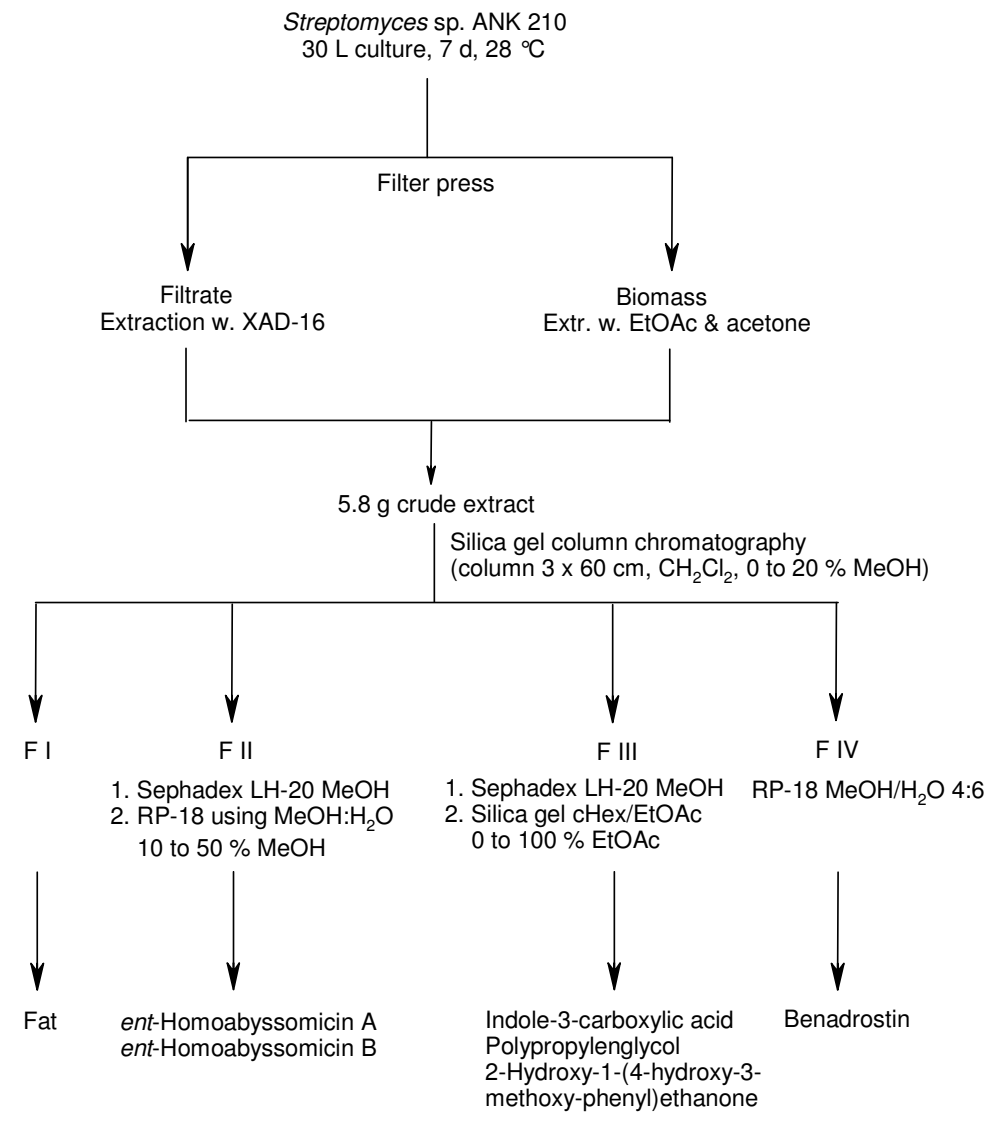

ent-Homoabyssomicin A (1): White crystals, UV absorbing at $254 \mathrm{~nm}, R_{\mathrm{f}}=0.45$ $\left(\mathrm{CH}_{2} \mathrm{Cl}_{2} / 5 \% \mathrm{MeOH}\right)$; a crystal suitable for single-crystal X-ray diffraction studies was obtained by slow evaporation of a solution in $\mathrm{CHCl}_{3} / 5 \% \mathrm{MeOH}$ at $4{ }^{\circ} \mathrm{C} ;[\alpha]_{\mathrm{D}}^{20}=$ -22.1 (c $\left.=0.117, \mathrm{CH}_{3} \mathrm{OH}\right)$; UV/VIS: $\lambda_{\max }(\log \varepsilon)=\left(\mathrm{CH}_{3} \mathrm{CN}\right): 276$ sh (2.80), 223 (2.93); IR $v_{\max }^{K B r} \mathrm{~cm}^{-1}: 3428,2928,1762,1726,1636,1460,1383,1250,1111,1075$, 1030; $\mathrm{CD}\left(\mathrm{c} 1.89 \times 10^{-4} \mathrm{M}, \mathrm{MeOH}, 22{ }^{\circ} \mathrm{C}\right): \lambda_{\max } 202 \mathrm{~nm}(\Delta \varepsilon 3.35) ;{ }^{1} \mathrm{H}$ NMR $(600$ $\mathrm{MHz}, \mathrm{MeOH})$ and ${ }^{13} \mathrm{C}$ NMR (125 MHz, MeOH); (+)-ESIMS: $m / z=379\left([\mathrm{M}+\mathrm{H}]^{+}\right.$, 90), $779\left([2 \mathrm{M}+\mathrm{Na}]^{+}, 50\right)$; (+)-HRESIMS: $m / z=379.17512[\mathrm{M}+\mathrm{H}]^{+}$, (calcd. for $\left[\mathrm{C}_{20} \mathrm{H}_{27} \mathrm{O}_{7}\right]^{+}, 379.17513$.

ent-Homoabyssomicin B (2): White solid, UV absorbing at $254 \mathrm{~nm}, R_{\mathrm{f}}=0.48$ $\left(\mathrm{CH}_{2} \mathrm{Cl}_{2} / 5 \% \mathrm{MeOH}\right) ;[\alpha]_{\mathrm{D}}^{20}=+41.7\left(\mathrm{c}=0.034, \mathrm{CH}_{3} \mathrm{OH}\right)$; UV/VIS: $\lambda_{\max }(\log \varepsilon)=$ $\left(\mathrm{CH}_{3} \mathrm{CN}\right): 287$ (3.10), 241 (3.56), 219 sh (3.51); IR $v_{\max }^{K B r} \mathrm{~cm}^{-1}: 3428,1742,1656$, 1454, 1396, 1020, 666; $\mathrm{CD}\left(\mathrm{c} 1.085 \times 10^{-4} \mathrm{M}, \mathrm{MeOH}, 22^{\circ} \mathrm{C}\right): \lambda_{\max }=207 \mathrm{~nm}(\Delta \varepsilon$ 14.57), $236 \mathrm{~nm}(\Delta \varepsilon 7.48) ;{ }^{1} \mathrm{H}$ NMR $(600 \mathrm{MHz}, \mathrm{MeOH})$ and ${ }^{13} \mathrm{C}$ NMR (125 MHz, 
$\mathrm{MeOH})$ data see table 1; (+)-ESIMS: $m / z=457\left([\mathrm{M}+\mathrm{Na}]^{+}, 100\right), 891\left([2 \mathrm{M}+\mathrm{Na}]^{+}\right.$, 50); (+)-HRESIMS: $m / z=457.14686[\mathrm{M}+\mathrm{Na}]^{+}$, (calcd. for $\left[\mathrm{C}_{22} \mathrm{H}_{26} \mathrm{O}_{9} \mathrm{Na}\right]^{+}$, 457.14690). 


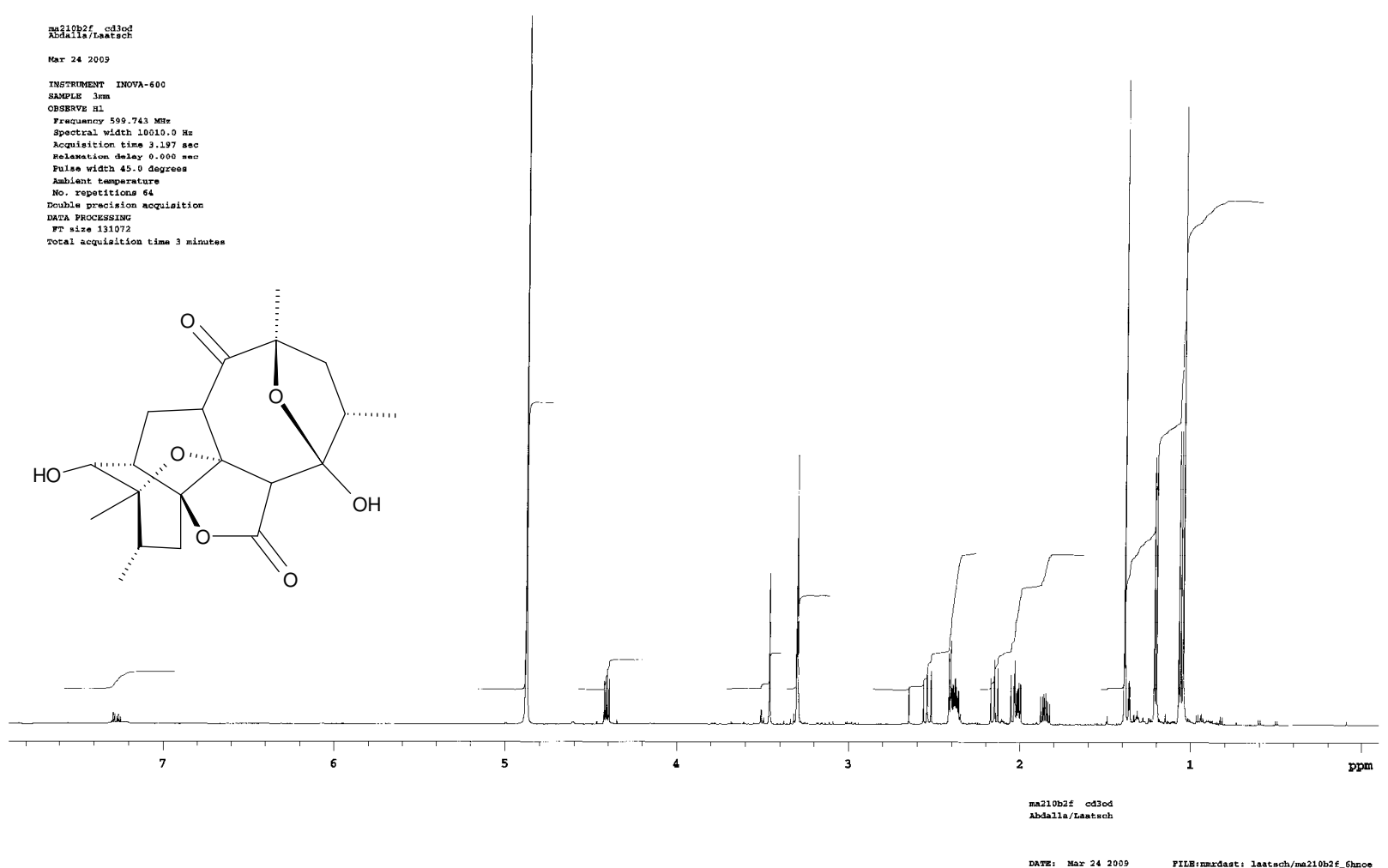

Figure S1: ${ }^{1} \mathrm{H}$ NMR spectrum (600 MHz, MeOD) of ent-homoabyssomicin A (1) 


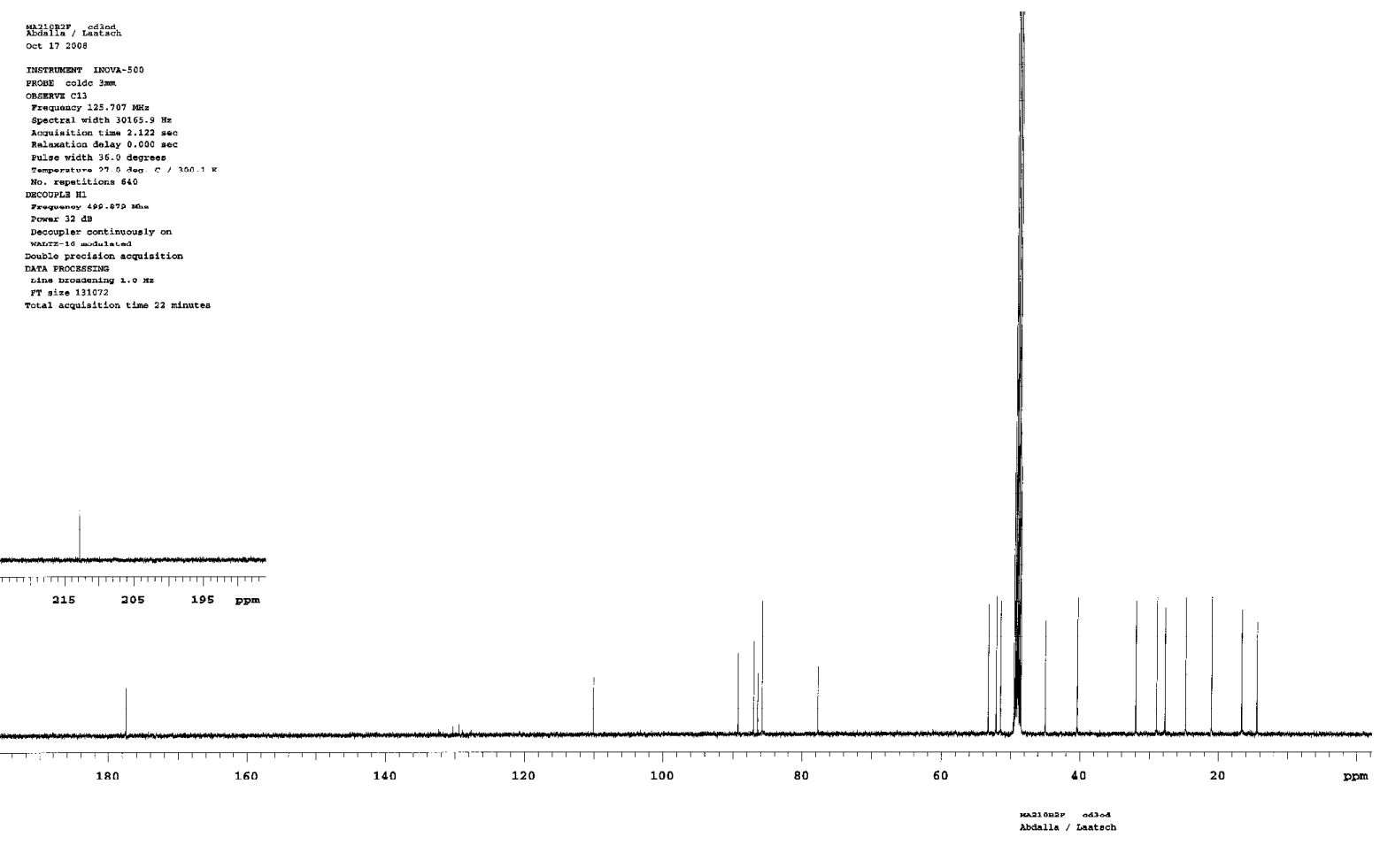

Figure S2: ${ }^{13} \mathrm{C}$ NMR spectrum (125 MHz, MeOD) of ent-homoabyssomicin A (1) 

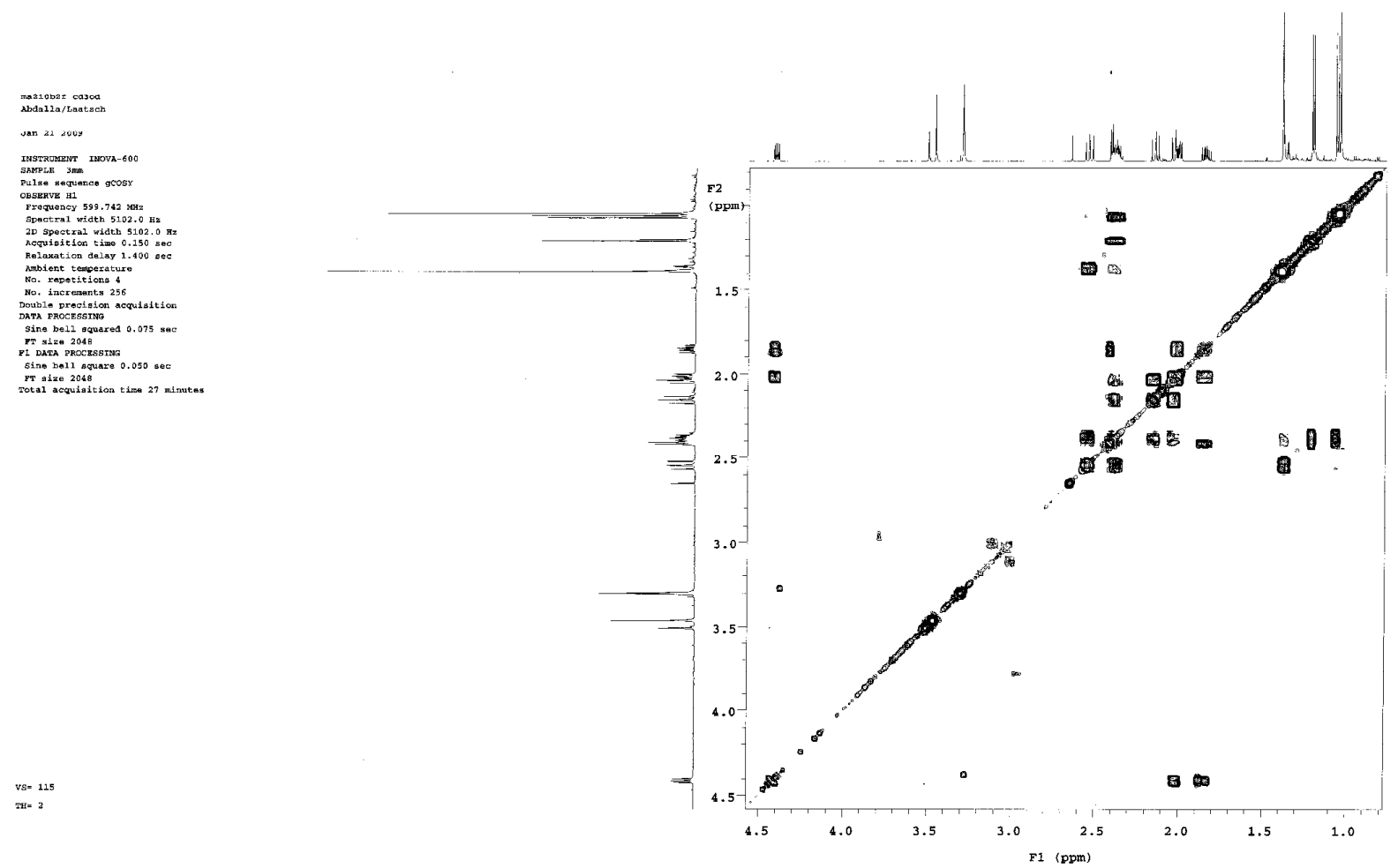

Figure S3: ${ }^{1} \mathrm{H},{ }^{1} \mathrm{H}$ COSY spectrum (600 MHz, MeOD) of ent-homoabyssomicin A (1) 
8

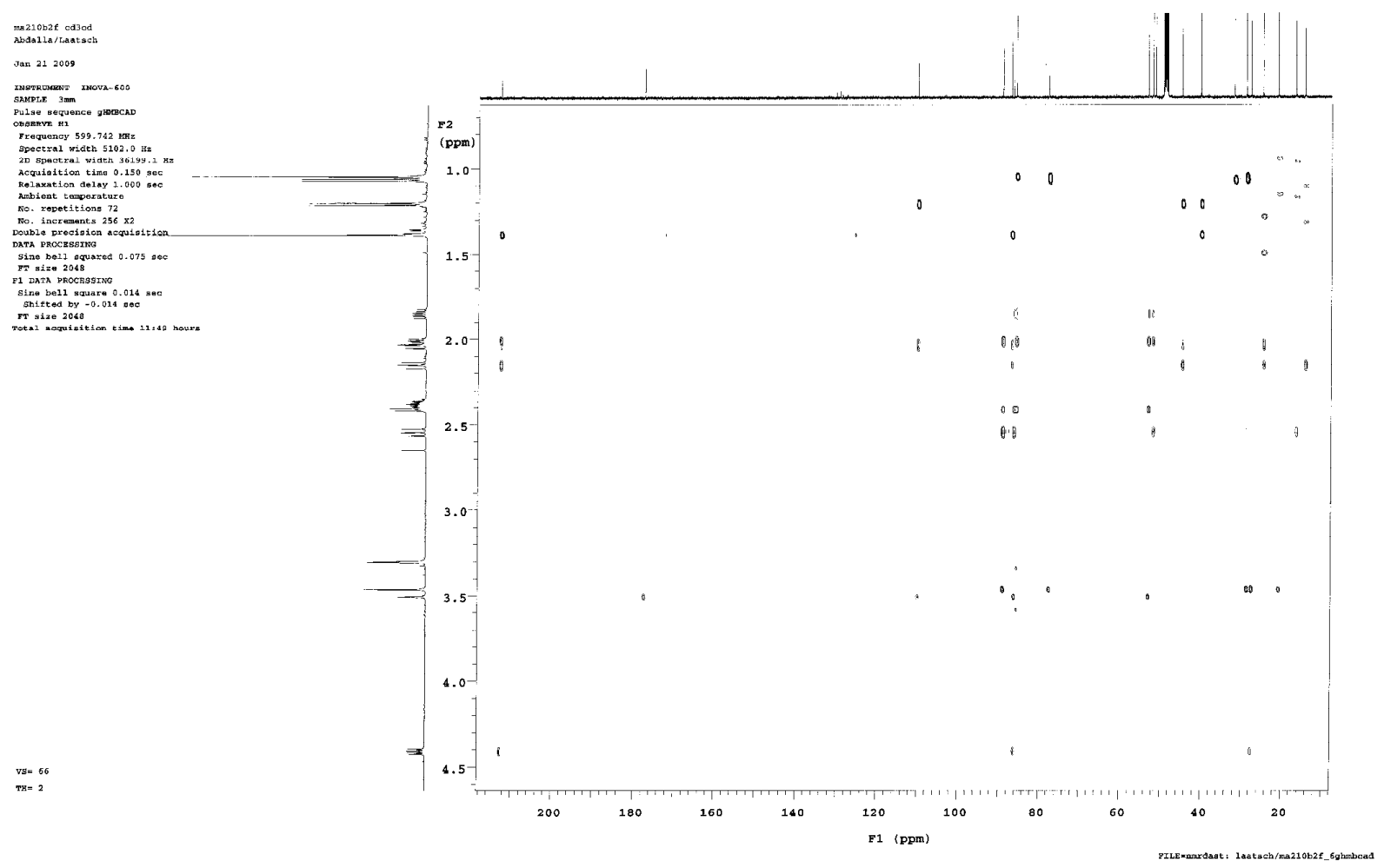

Figure S4: HMBC spectrum (600 MHz, MeOD) of ent-homoabyssomicin A (1) 


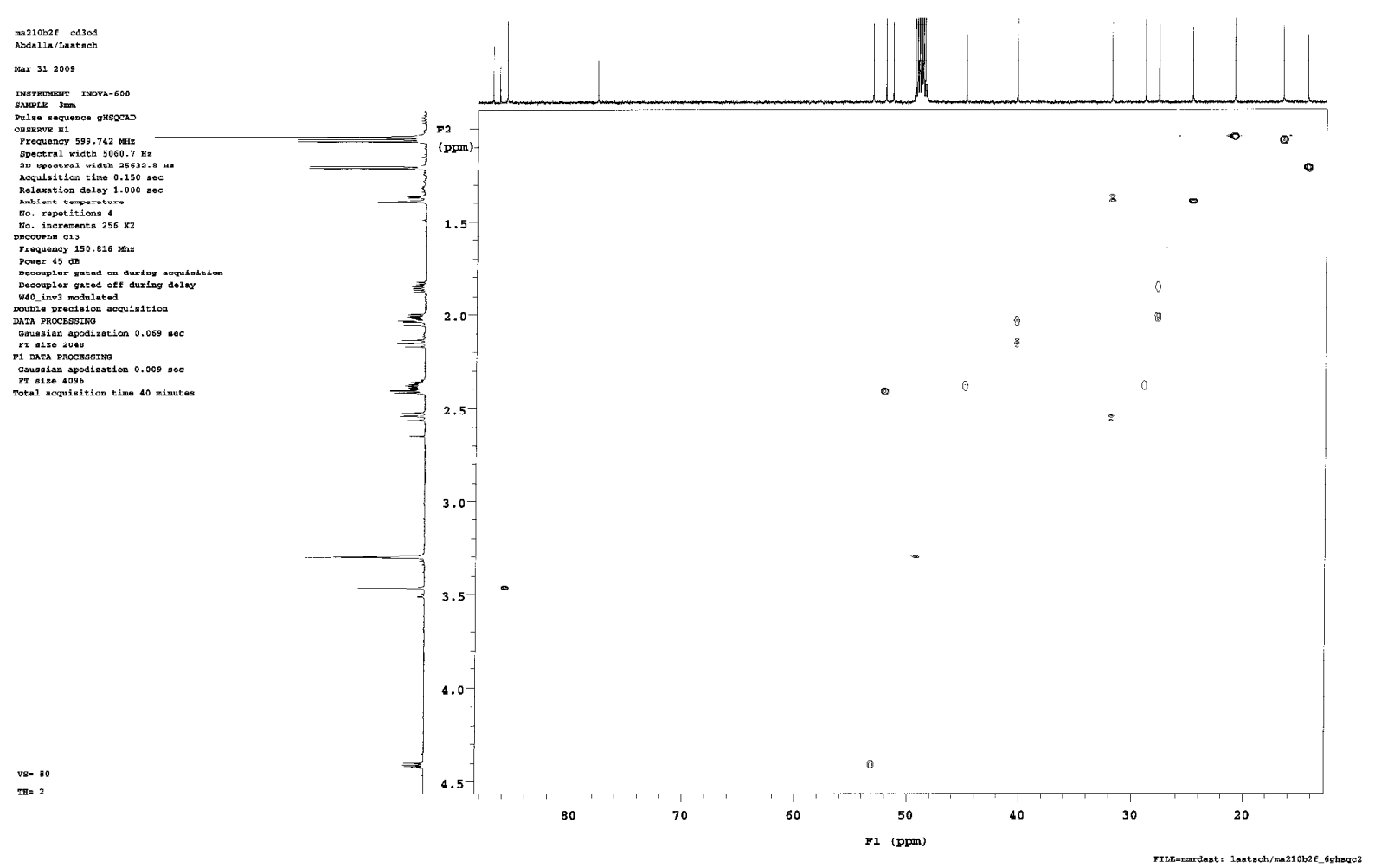

Figure S5: HSQC spectrum (600 MHz, MeOD) of ent-homoabyssomicin A (1) 


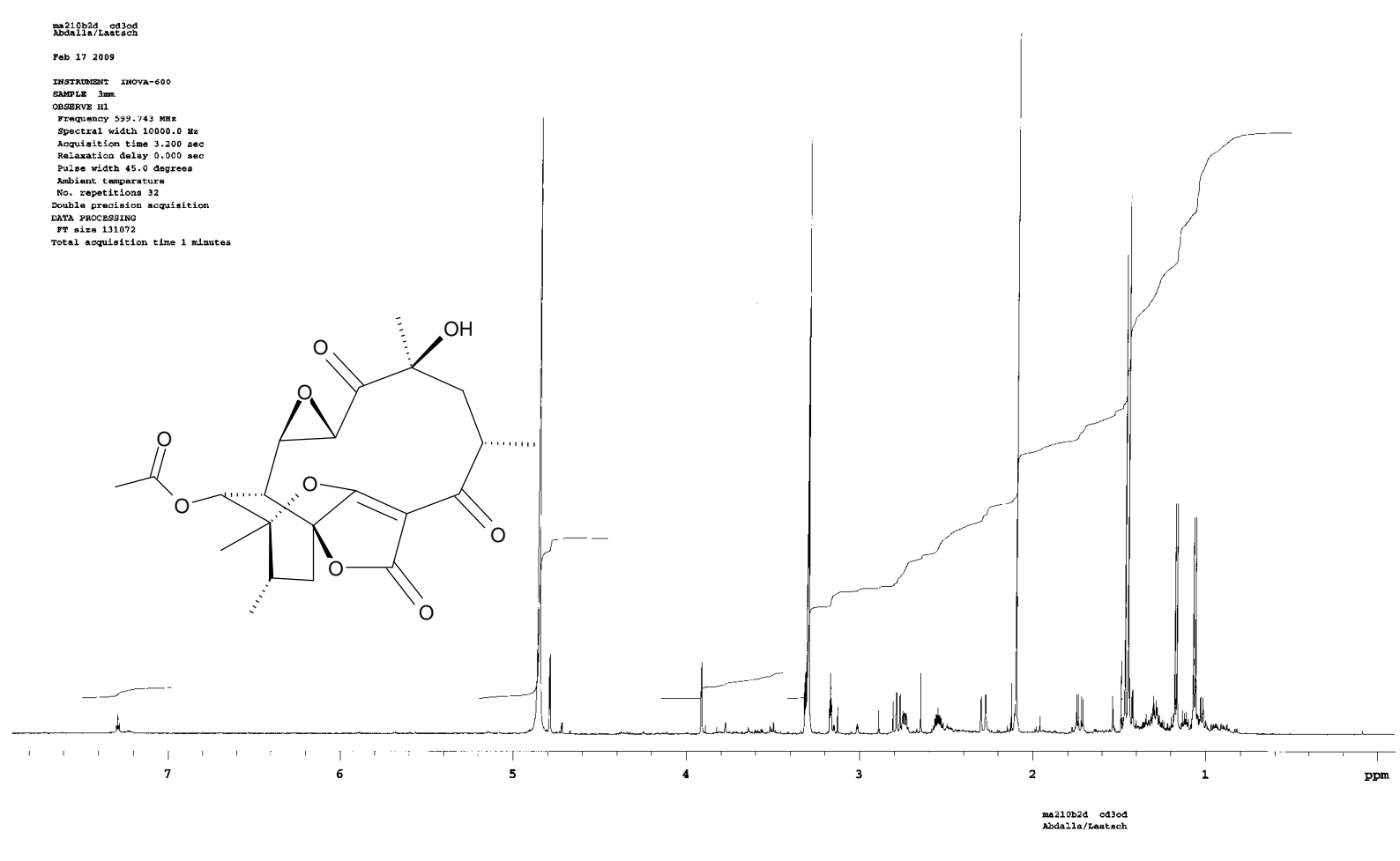

Figure S6: ${ }^{1} \mathrm{H}$ NMR spectrum (600 MHz, MeOD) of ent-homoabyssomicin B (2) 


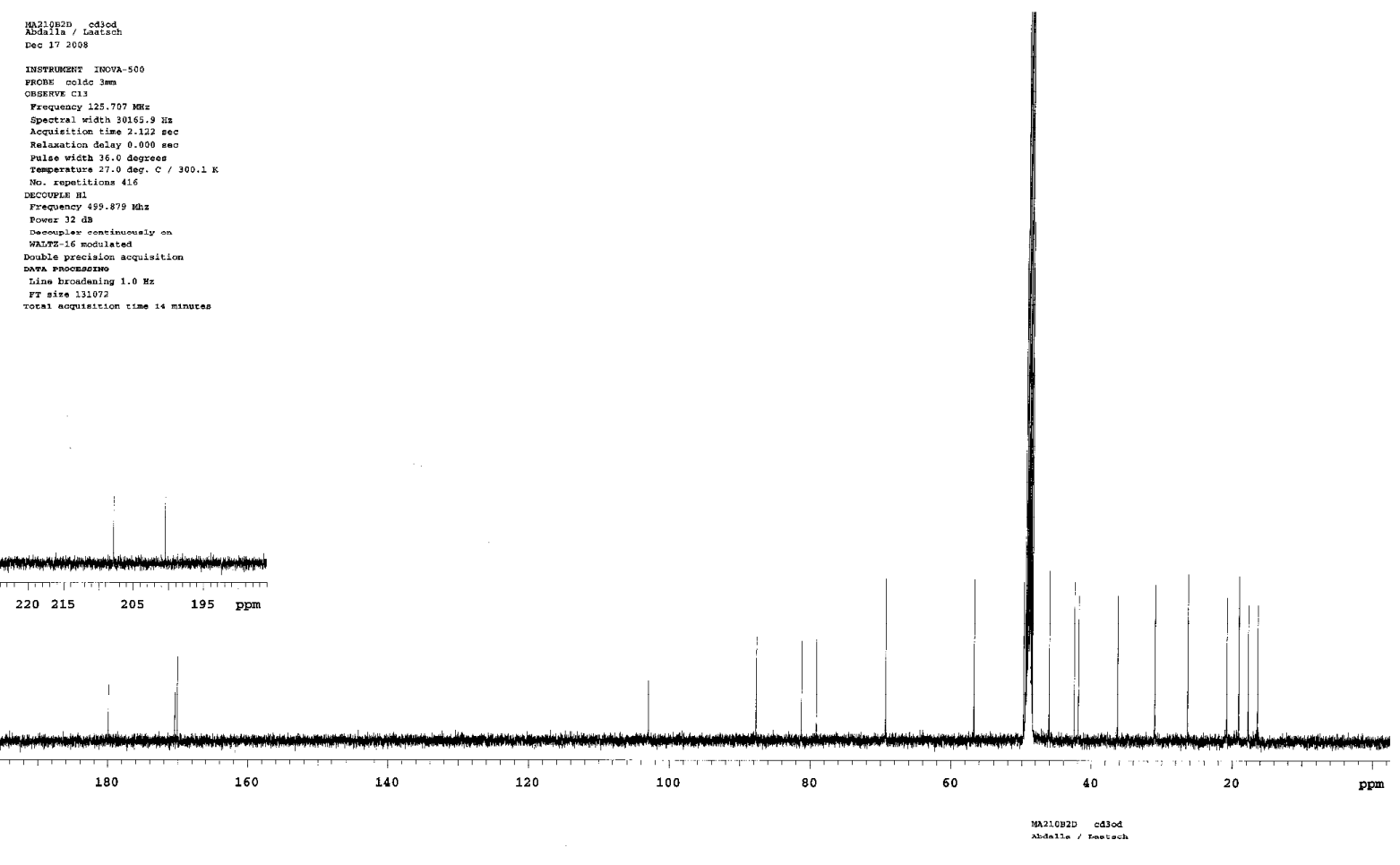

Figure S7: ${ }^{13} \mathrm{C}$ NMR spectrum (125 MHz, MeOD) of ent-homoabyssomicin B (2) 


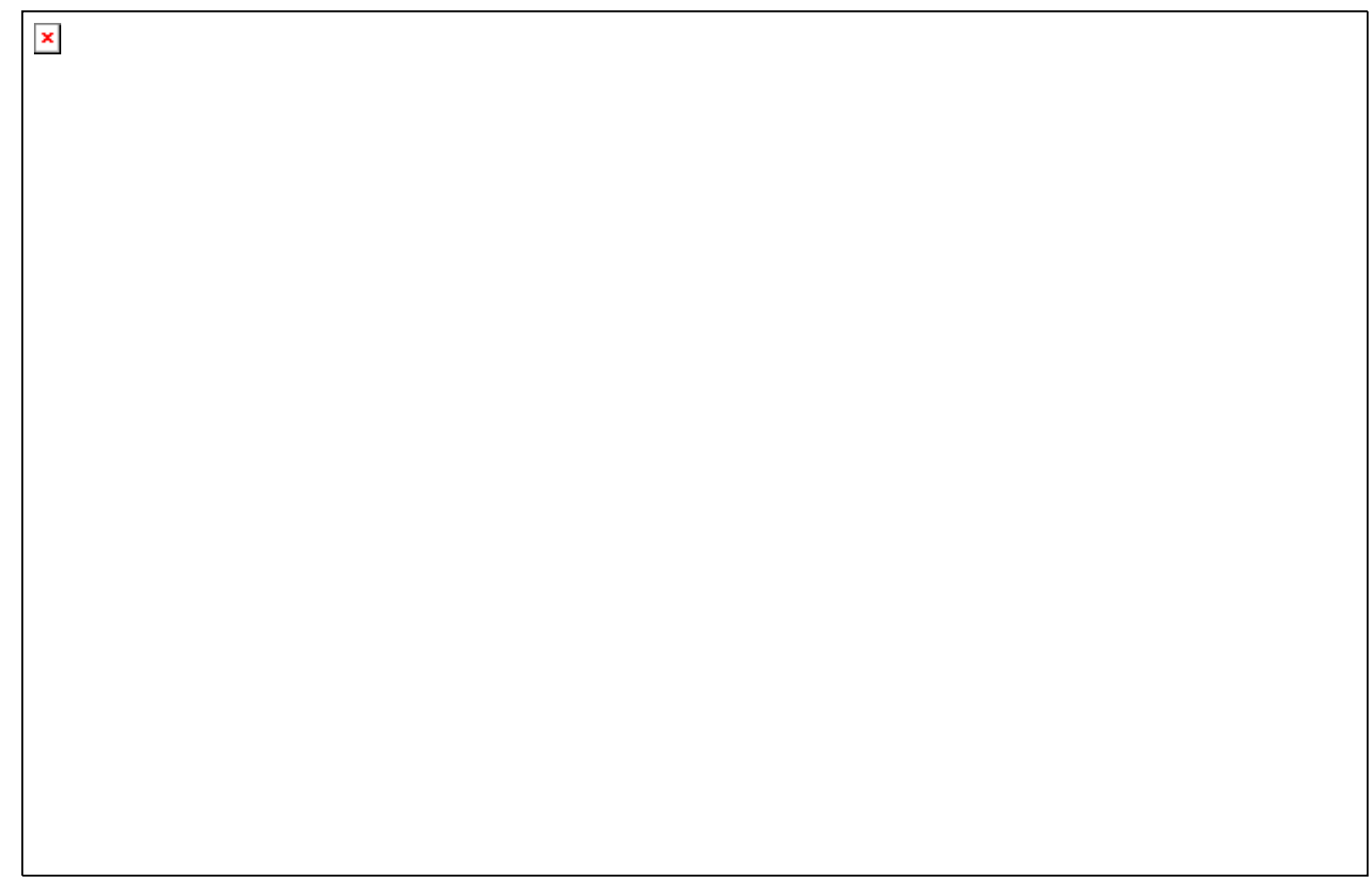

Figure S8: ${ }^{1} \mathrm{H},{ }^{1} \mathrm{H}$ COSY spectrum (600 MHz, MeOD) of ent-homoabyssomicin B (2) 


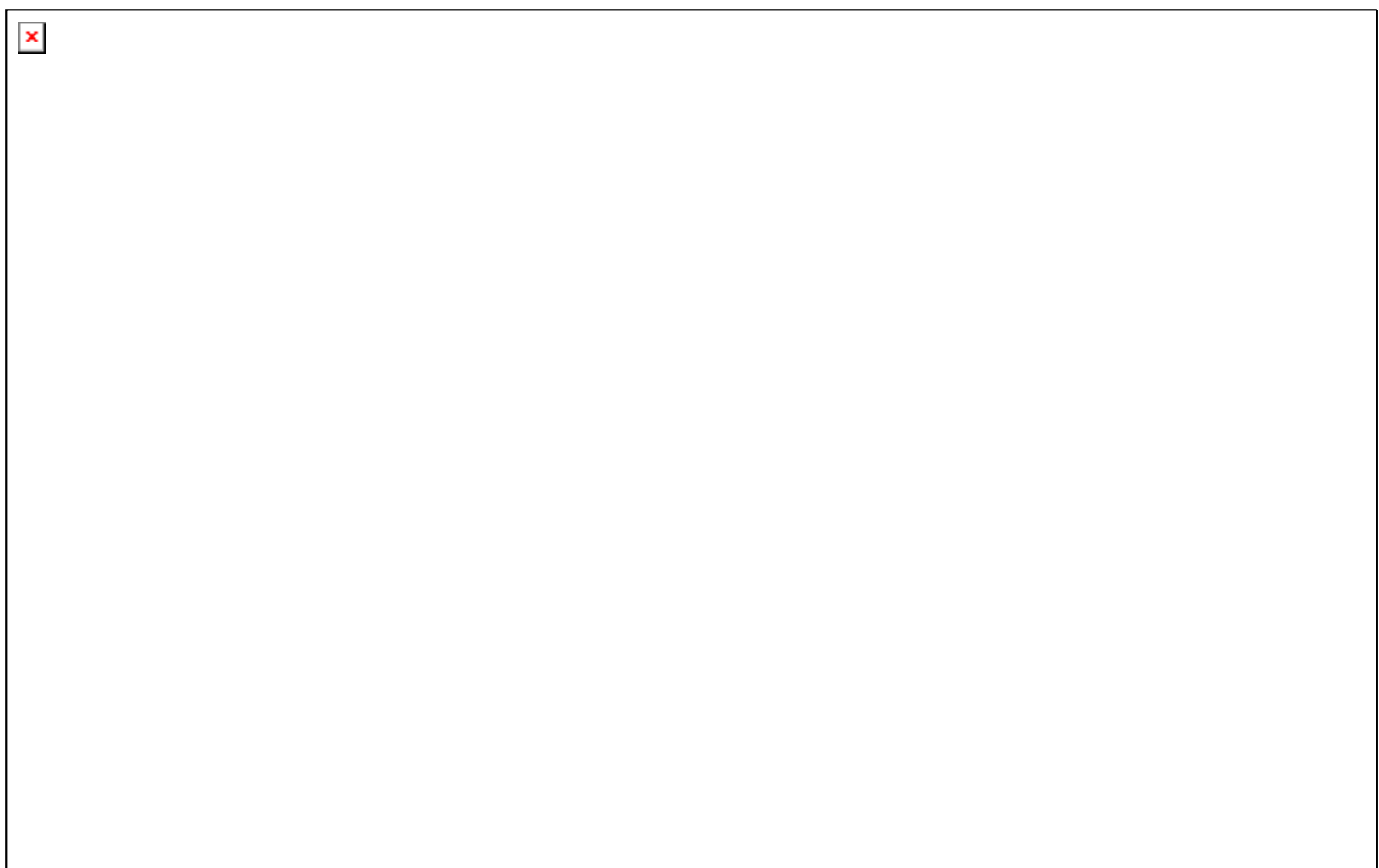

Figure S9: HMBC spectrum (600 MHz, MeOD) of ent-homoabyssomicin B (2) 


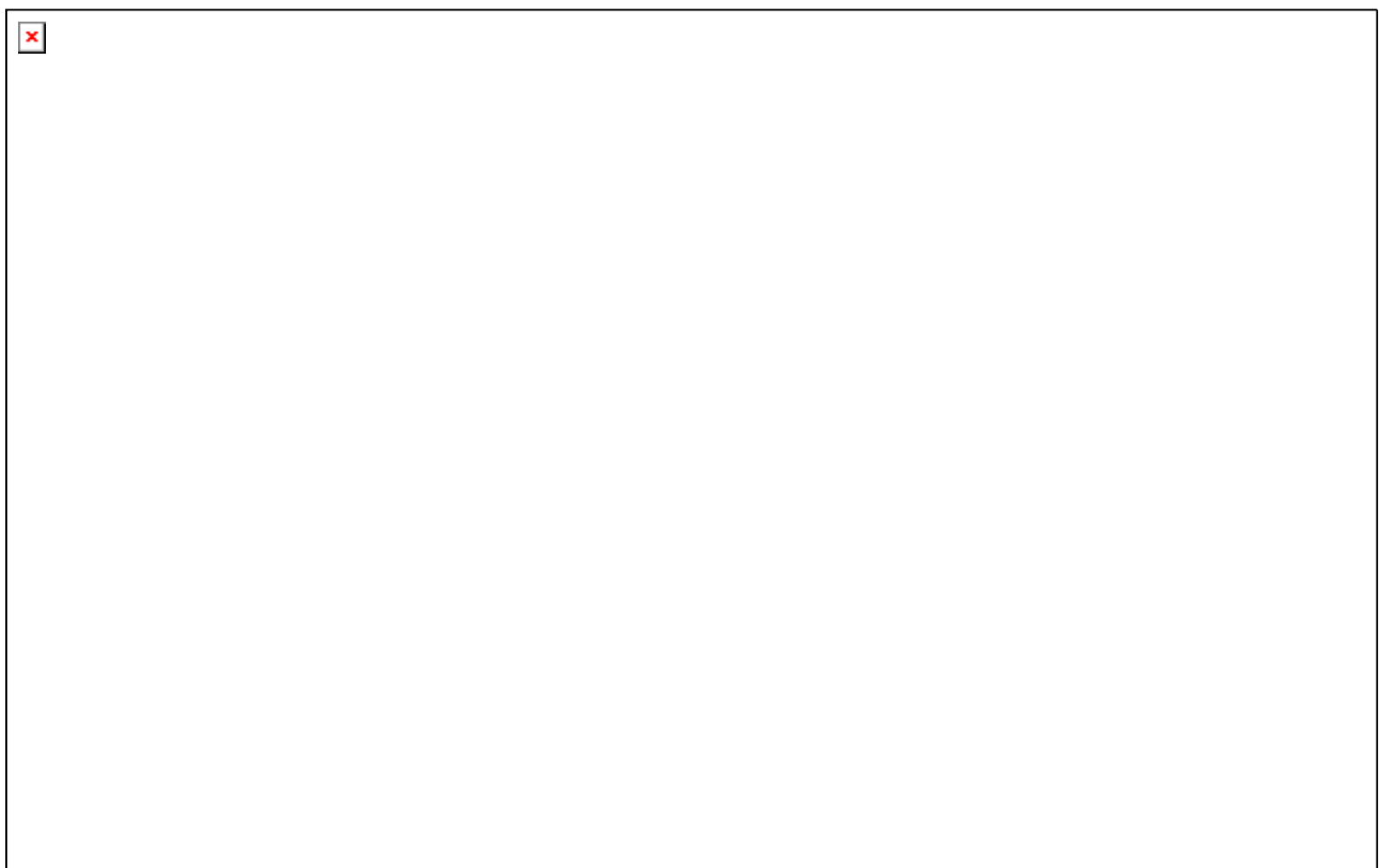

Figure S10: HSQC spectrum (600 MHz, MeOD) of ent-homoabyssomicin B (2) 
Crystal Data for ent-Homoabyssomicin A (1)

Formula

Formula Weight

Crystal System

Space group

a, b, c [A]

$\mathrm{V}\left[\AA^{3}\right]$

Z

$\mathrm{D}$ (calc) $\left[\mathrm{g} / \mathrm{cm}^{3}\right]$

$\mathrm{Mu}\left(\mathrm{CuK}_{\alpha}\right)[/ \mathrm{mm}]$

$\mathrm{F}(000)$

Crystal Size [mm]

\section{Data Collection}

Temperature $(\mathrm{K})$

Radiation [̊̊]

$\Theta$ Min-Max [Deg]

Dataset $\left(\mathrm{h}_{\min }: \mathrm{h}_{\max } ; \mathrm{k}_{\min }: \mathrm{k}_{\max } ; \mathrm{l}_{\min }, \mathrm{l}_{\max }\right)$

Tot., Uniq. Data, R(int)

Observed data $[\mathrm{I}>0.0 \sigma(\mathrm{I})]$

\section{Refinement}

$\mathrm{N}_{\text {ref }}, \mathrm{N}_{\text {par }}$

R, wR2, S

Weighting scheme

Max. and Av. Shift/Error

Flack x

Min. and Max. Resd. Dens. [e/ $\left.\AA^{3}\right]$
$\mathrm{C}_{20} \mathrm{H}_{26} \mathrm{O}_{7}, \mathrm{H}_{2} \mathrm{O}$

396.42

orthorhombic

$\mathrm{P} 2{ }_{1} 2_{1} 2_{1}$ (No. 19)

7.579(1), 15.663(2), 16.461(3)

1954.1(5)

4

1.347

0.870

848

$0.02 \times 0.09 \times 0.15$

100

CuKa, 1.54180

$3.9,72.3$

-9: 9; -19:18; -15:19

19265, 3581, 0.073

2653

2653, 366

$0.0314,0.0292,1.30$

$\mathrm{w}=1 /\left[\sigma^{2}\left(\mathrm{~F}_{\mathrm{o}}\right)\right]$

$0.00,0.00$

0.06(19)

$-0.19,0.17$ 